University of Nebraska - Lincoln

DigitalCommons@University of Nebraska - Lincoln

$1-1-2008$

\title{
Self-Assembly of a Micrometers-Long One-Dimensional Network of Cemented Au Nanoparticles
}

\author{
Vivek C. Maheshwari \\ University of Nebraska-Lincoln, vmaheshw@uwaterloo.ca \\ Jennifer Kane \\ University of Nebraska - Lincoln \\ Ravi F. Saraf \\ University of Nebraska-Lincoln, rsaraf2@unl.edu
}

Follow this and additional works at: https://digitalcommons.unl.edu/cbmesaraf

Part of the Biomechanics and Biotransport Commons

Maheshwari, Vivek C.; Kane, Jennifer; and Saraf, Ravi F., "Self-Assembly of a Micrometers-Long OneDimensional Network of Cemented Au Nanoparticles" (2008). Ravi Saraf Publications. 1.

https://digitalcommons.unl.edu/cbmesaraf/1

This Article is brought to you for free and open access by the Chemical and Biomolecular Research Papers -- Faculty Authors Series at DigitalCommons@University of Nebraska - Lincoln. It has been accepted for inclusion in Ravi Saraf Publications by an authorized administrator of DigitalCommons@University of Nebraska - Lincoln. 


\title{
Self-Assembly of a Micrometers-Long One-Dimensional Network of Cemented Au Nanoparticles
}

\author{
Vivek Maheshwari, Jennifer Kane, and Ravi F. Saraf* \\ Department of Chemical and Biomolecular Engineering, University of Nebraska-Lincoln, Lincoln, NE 68588 USA \\ * Correspondence email: rsaraf@unlnotes.unl.edu
}

\begin{abstract}
One-dimensional Au nanoparticles connected by ionic linkers self-assemble into a micrometer-scale network. Reactions between linker ions reinforce the assembly and lead to the formation of a continuous network. This continuous network demonstrates roomtemperature Coulomb-blockade characteristics that are indicative of electron transport along the 1D pathways.
\end{abstract}

One-dimensional (1D) nanostructures are good candidate materials for use in electronic devices because they can act as both nanodevices and circuitry, allowing the integration of power sources and extraction of signals. Basic logic circuits, ${ }^{[1]}$ a variety of chemical and biochemical sensors, ${ }^{[2]}$ and devices with neural cell networks ${ }^{[3]}$ have been produced using carbon nanotubes and nanowires in which the 1D nanostructure forms the wiring material as well as the device. ${ }^{[4]}$ The use of necklaces of nanoparticles provides a highly versatile route for the production of 1D nanostructures in which both the chemistry of the nanoparticle and its diameter can be tailored. Necklaces of nanoparticles have been assembled using chains of DNA, ${ }^{[5]}$ nanofibrils, ${ }^{[6]}$ block copolymers, ${ }^{[7]}$ functionalized ligands, ${ }^{[8]}$ and polyelectrolytes ${ }^{[9,10]}$ as scaffolds. In addition to scaffolddirected self-assembly, electric-dipole interactions between nanoparticles can also be used to make $1 \mathrm{D}$ necklaces. ${ }^{[11,12]}$ However, in each of these structures there is no special interconnection between the particles beyond the physical contact that is mediated by the morphology of the scaffold. In this report, we demonstrate the self-assembly of a necklace of $\mathrm{Au}$ nanoparticles on a flexible polymer chain that is mediated by a linker ion that is subsequently used to cement the particles together with an inorganic material. The diameter of the $\mathrm{Au}$ nanoparticles was $10 \mathrm{~nm}$, the polymer was poly(styrene sulfonate) (PSS) with a molecular weight of $5 \times 10^{5} \mathrm{Da}$, and the cementing material was $\mathrm{CdS}$. The cementing process could be directly observed using transmission electron microscopy (TEM). After the cementing process, chains of individual $\mathrm{Au}$ nanoparticles had been transformed into wirelike structures with widths (i.e., diameters) of $\sim 10 \mathrm{~nm}$.

A $1 \mathrm{mg} \mathrm{mL}-1$ cadmium perchlorate hydrate solution $(\mathrm{Cd}$ solution) was added dropwise, under constant stirring, to a citrate-functionalized 10-nm-Au-nanoparticle solution until a volume ratio of $\mathrm{Cd}$ solution:Au-nanoparticle solution of 1:4 was obtained. The wine-red color of the pure Au solution had a surface plasmon resonance (SPR) absorption band at $\sim 525 \mathrm{~nm}$ (Au curve in Figure 1). ${ }^{[13]}$ On the other hand, after
$12 \mathrm{hrs}$ of stirring the $\mathrm{Cd}-\mathrm{Au}$ solution was a violet-blue color and its SPR band was red-shifted to $\sim 620 \mathrm{~nm}$ with a shoulder at $525 \mathrm{~nm}$ (Au-Cd curve in Figure 1). This red shift is associated with 1D assembly of Au nanoparticles and indicated delocalization of the plasmon wave due to coupling between closely spaced particles. ${ }^{[8,11]}$ The assembly had clustered particles that formed a stringlike morphology (Figure S1, Supporting Information). This coupling between the nanoparticles occurred because the divalent $\mathrm{Cd}^{2+}$ ions interacted with negatively charged carboxylic acid groups from the citrate ions on adjacent $\mathrm{Au}$ nanoparticles to form interparticle "bridges.",[14] Reducing the $\mathrm{Cd}$ solution: $\mathrm{Au}$ nanoparticle solution ratio to $0.5: 4$, reduced the number of $\mathrm{Cd}^{2+}$ ions per bridge and led to a smaller shift in the SPR band to $\sim 550 \mathrm{~nm}$ (PSS-Cd-Au 0.5 curve in Figure 1). For ratios larger than 1:4, the Au particles precipitated out. Furthermore, as the TEM analysis suggests very few isolated Au particles (e.g., Figures S1 and S2), the shoulder at $525 \mathrm{~nm}$ could be attributed to electron plasmon motion along the shorter 10-nm axis of the 1D assembly.

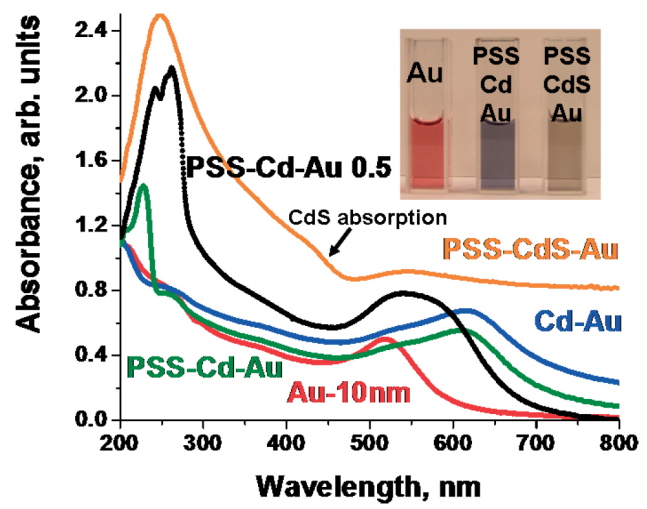

Figure 1. Absorption spectra of the assembly at each stage of the transformation. For clarity, the PSS-CdS-Au spectrum has been shifted vertically by 0.5 units. The change in the color of the solution, from wine red to violet blue and then muddy yellow, is visible in the photograph. 
To guide the bridged cluster into a long-range 1D assembly, $200 \mu \mathrm{L}$ of PSS solution $\left(1 \mathrm{mg} \mathrm{mL}^{-1}\right)$ was added to $5 \mathrm{~mL}$ of $\mathrm{Cd}-\mathrm{Au}$ solution. The PSS sulfonate groups preferred to attach to the free $\mathrm{Cd}$ ions ${ }^{[10]}$ on the nanoparticle surface that were not involved in particle linkages. In contrast to the $\mathrm{Au}-$ $\mathrm{Cd}$ suspension stability (less than 2 weeks), the PSS-Cd-Au solution was remarkably stable and showed no change in color or in the position of the SPR band (indicative of agglomeration) over a time period of more than 6 months. This increase in stability was attributed to the steric and electrostatic hindrance that arises from the anionic PSS. The only significant change was the appearance of the PSS absorbance band at $\sim 265 \mathrm{~nm}$ (Figure 1). The 1D necklace of Au nanoparticles that was deposited on the PSS chain was clearly visible in the TEM image (Figure 2a). The arrangement of the particles was fairly discrete, with an interparticle distance of 1-2 nm. The addition of $\mathrm{Na}_{2} \mathrm{~S}$ to the solution, at a ratio 0.7 times the stoichiometric ratio with respect to $\mathrm{Cd}$, changed the absorbance spectrum significantly (PSS-CdS-Au curve in Figure 1). The SPR band of Au was evident from the weak peak at $540 \mathrm{~nm}$ that extended to $800 \mathrm{~nm}$.

The shift in the SPR peak from $\sim 620 \mathrm{~nm}$ back to $\sim 540 \mathrm{~nm}$ for the Au particles (Au-10-nm curve in Figure 1) indicated weaker plasmon coupling resulting from the higher dielectric constant of CdS compared to that of the ionic bridge. A shoulder at $415 \mathrm{~nm}$ that was highly blue shifted compared to bulk $\mathrm{CdS}(510 \mathrm{~nm})$ was attributed to formation of interparticle CdS (cement) (PSS-CdS-Au curve in Figure 1). The formation of $\mathrm{CdS}$ cement was more evident in the photoluminescence (PL) spectrum (Figure 3 ). Figure 3 shows a single emission peak at $\sim 455 \mathrm{~nm}$ for the Au particle necklace on PSS both before (inset in Figure 3) and after cementing (i.e., for $\mathrm{Au}-\mathrm{Cd}-\mathrm{PSS}$ and $\mathrm{Au}-\mathrm{CdS}-\mathrm{PSS}$, respectively). This $455 \mathrm{~nm}$ peak arose from radiative recombination of electrons and holes in the interband transition for the Au nanoparticles. ${ }^{[13,15]}$ However, the spectrum of the cemented necklace (Au-CdS-PSS) had an emission band centered at $\sim 610 \mathrm{~nm}$, in addition to the $455 \mathrm{~nm}$ peak. This new $610 \mathrm{~nm}$ band was associated with the formation of CdS nanoscale cement between Au particles. The larger red shift in photoluminescence for CdS cement at $610 \mathrm{~nm}$ than for pure CdS at $530 \mathrm{~nm}^{[16]}$ was attributed to the change in surface states of $\mathrm{CdS}$ due to the interface with $\mathrm{Au}$. The exothermic reaction of $\mathrm{Na}_{2} \mathrm{~S}$ and the affinity of the gold surface for sulfur led to the formation of a "metallurgical" junction and a small amount of diffusion of $\mathrm{Au}$ into the $\mathrm{CdS}$ cement. The assignment of the $610 \mathrm{~nm}$ band to an impurity energy level from $\mathrm{Au}$ in the bandgap of $\mathrm{CdS}$ (i.e., $\mathrm{CdS}-\mathrm{Au}$ ) is consistent with reports in the literature. ${ }^{[17]}$

The cementing of the Au nanoparticles into the necklace, due to addition of $\mathrm{Na}_{2} \mathrm{~S}$, was also observed by TEM (Figure $2 b$ ). In Figure $2 b$, we see that the discreet particle necklace (Figure 2a) was transformed into a continuous wire where silhouettes of the nanoparticles appear as nodules in the wire. The width of the wire was $\sim 10 \mathrm{~nm}$, which indicated that it was composed of a 1D row of particles. The morphology of the necklace, characterized by branches and loops, appeared to remain intact. Also, the length of the clusters remained approximately the same, which indicated that the necklace was fairly robust; it did not fracture during processing, for example when making the TEM samples. The fact that topology of the necklace did not change significantly on cementing suggested that the necklace was a 3D network of 1D necklaces. In some regions, the wire appeared to have agglomerations with widths of $\sim 20 \mathrm{~nm}$ or larger that seem to be remnants of entanglements that were observed in the precemented necklace seen in Figure 2a. Elemental analysis by energy dispersive Xray (EDX) with a $\sim 60 \mathrm{~nm}$ beam spot indicated the presence of CdS in the necklace (Figure S7). On cementing, the neck-

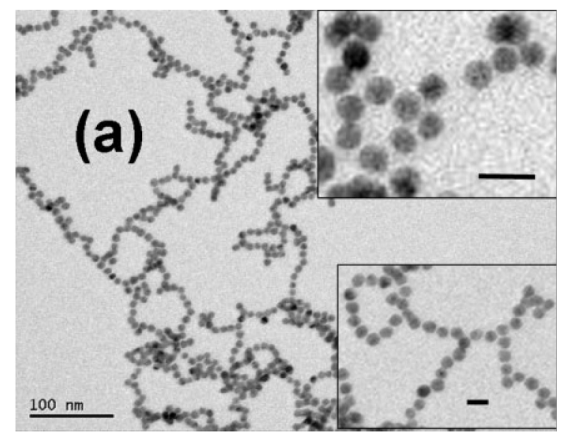

(c)

Figure 2. TEM and electrical analysis of the 1D branched assemblies. a) Discreet Au nanoparticle assembly resulting from the PSS-Cd-Au interaction. The inset scale bars are $20 \mathrm{~nm}$. b) On addition of $\mathrm{Na}_{2} \mathrm{~S}$, the discreet nanoparticle assembly is transformed to a continuous structure. c) Current (I) in response to applied bias $(V)$ for the necklace network between Au electrodes spaced $50 \mu \mathrm{m}$ apart as a function of temperature. d) Differential conductivity corresponding to the response in (c).

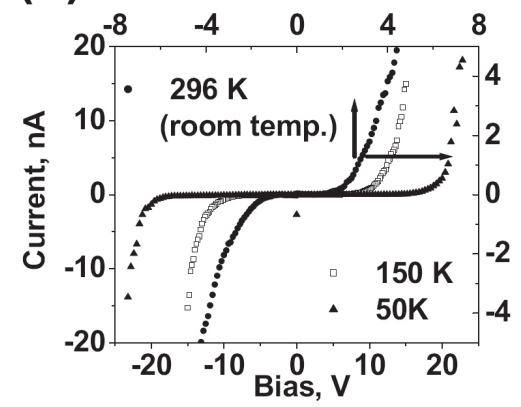

(d)

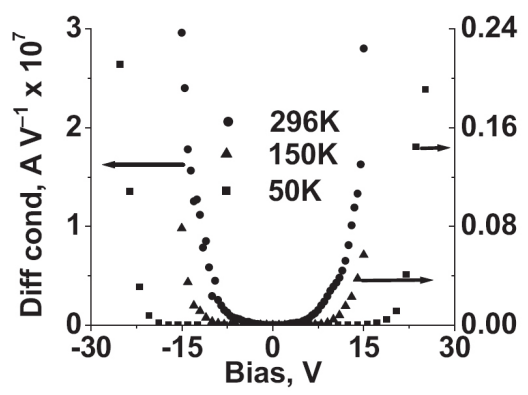




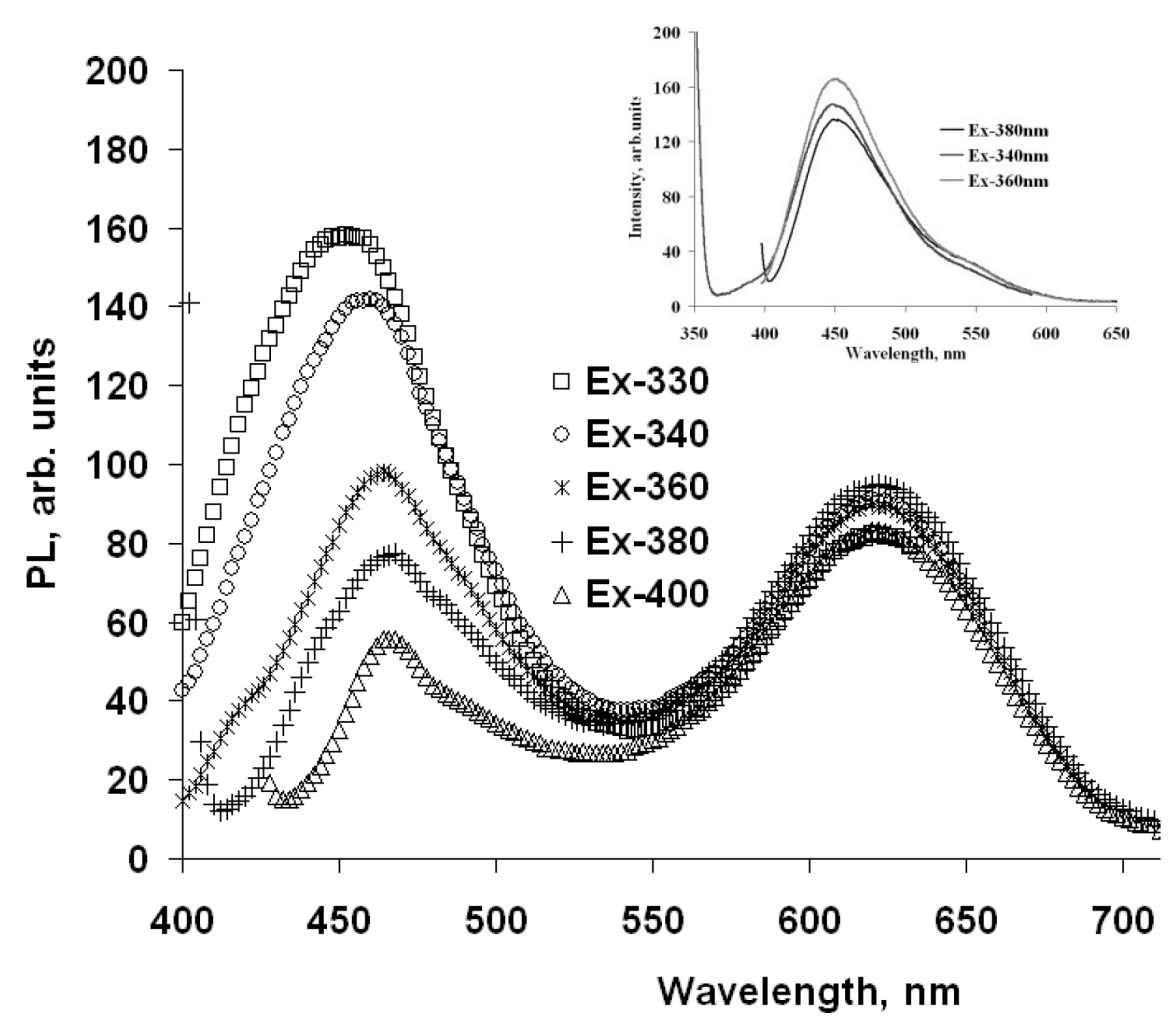

Figure 3. PL emission spectra of PSS-CdS$\mathrm{Au}$ and PSS-Cd-Au (inset). Both PL spectra show an emission peak at $\sim 455 \mathrm{~nm}$ due to $\mathrm{Au}$. An additional $620 \mathrm{~nm}$ peak, due to the formation of CdS cement, appears in the spectrum for PSS-CdS-Au.

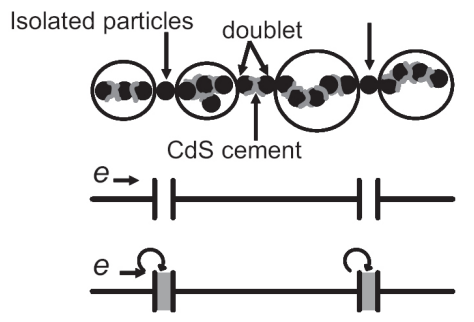

Figure 4. Schematic model of the necklace. Larger clusters are shown in circles are cemented together with CdS. The necklace is punctuated with isolated islands of singlets, doublets, and other short chains. lace became conducting with nonlinear current-voltage (I$V$ ) characteristics and a significant threshold bias $V_{\mathrm{T}}$ required for current flow. (Figure 2c). This $V_{\mathrm{T}}$ was indicative of a notable coulomb blockade effect at $296 \mathrm{~K}$ that increased at lower temperatures (Figure 2c-d). This observation was consistent with the $1 \mathrm{D}$ electron transport that is expected from the necklace morphology. ${ }^{[18]}$ Bias, $V>40 \mathrm{~V}$ led to unstable currents and eventually to a loss of conductivity. The cause of this irreversible collapse was local resistive heating of the structure. Therefore, the bias was limited to a maximum value of $25 \mathrm{~V}$ during current-voltage measurements because this meant the structure was stable for several days.

The behavior shown in Figure $2 \mathrm{c}-\mathrm{d}$ indicates an increase in $V_{\mathrm{T}}$ from $\sim 1.8 \mathrm{~V}$ to $\sim 15 \mathrm{~V}$ as the temperature is reduced from room temperature (RT) to $50 \mathrm{~K}$. The increase in $V_{\mathrm{T}}$ is not consistent with classical coulomb blockade behavior, which predicts no significant variation in $V_{\mathrm{T}}$ with temperature ${ }^{[19]}$ except for small effects such as those from the narrowing of the Fermi-Dirac distribution that affects electron tunneling. ${ }^{[20]}$ Assuming a dielectric constant of $\varepsilon=3$ for the medium surrounding the nanoparticle, for a device with a single isolated particle of diameter $d=10 \mathrm{~nm}$, the estimated bias $V_{\mathrm{T}}=e / 2 C=$ $e /\left(4 \pi \varepsilon \varepsilon_{0} d\right)$ is $\sim 50 \mathrm{mV}$ corresponding to a barrier energy of 50 meV (i.e., $2 k T$ at RT). Here, $C$ is the capacitance of the particle, $\varepsilon$ is the charge of an electron, and $\varepsilon_{0}$ is the electric permittivity in vacuum. This increase in $V_{\mathrm{T}}$ and the thermal behavior can both be explained by modeling the necklace as a percolating structure punctuated with nanoparticle islands made of one (singlet), two (doublet), or more particles (Figure 4). These islands can be thought of as defects resulting from CdS cement breaking off or as gaps, left by chance, that were too difficult for the cement to fill during the assembly process. The isolated particles are coupled to the percolating strands by a tunnel junction that is similar to a classical coulomb blockade setup. Thus, the large percolating clusters act as wires and the islands act as devices.

At RT only the "single" particle clusters (i.e., singlets) will contribute to the blockade voltage. As the isolated particles are in series the net capacitance $C_{\mathrm{T}}$ is

$$
\frac{1}{C_{\mathrm{T}}}=\frac{1}{C}+\frac{1}{C}+\ldots . .=\frac{n}{C}=\frac{n}{2 \pi \varepsilon \varepsilon_{0} d}
$$

where $n$ is the number of clusters. Thus, the effective size of particle is $d / n$, leading to a coulomb blockade voltage, $V_{\mathrm{T}}=$ $e / 2 C_{\mathrm{T}}=n(e / 2 C)=n V_{1}$ (where $V_{1}$ is the coulomb blockade for a single nanoparticle). Thus, an increase in $V_{\mathrm{T}}$ from $50 \mathrm{mV}$ for single particle to $1.8 \mathrm{~V}$ for the necklace at $\mathrm{RT}$ will require an estimated $n$ of $\sim 35$. In other words, on average an electron transported over the $50 \mathrm{~lm}$ gap will encounter only 35 isolated defects.

Reducing the temperature (i.e., lowering $k T$ ) allows larger clusters to cause coulomb blockades. The capacitance continues to be reduced as more terms corresponding to larger clusters are added to Equation 1 and this leads to a larger blockade voltage, $V_{\mathrm{T}}$. Because $k T$ is reduced from $25 \mathrm{meV}$ (at RT) to $\sim 4.3 \mathrm{meV}$ at $50 \mathrm{~K}$, a cluster of $\sim 5$ particles can act as a blockade. Furthermore, the distribution of cluster sizes (singlet, double, etc.) cause the coulomb blockade $V_{\mathrm{T}}$ to become broader. A similar thermal effect on $V_{\mathrm{T}}$ has been observed for a broad distribution of $\mathrm{Au}$ islands in a one-dimensional arrangement ${ }^{[18]}$ and in a two-dimensional array. ${ }^{[21]}$

The role of $\mathrm{Cd}^{2+}$ in the bridging of $\mathrm{Au}$ nanoparticles and their subsequent cementing by the formation of $\mathrm{CdS}$ is confirmed by control experiments. TEM and optical spectroscopy were used to confirm that replacing $\mathrm{Cd}^{2+}$ salt with a similar concentration of monovalent salt, such as sodium chloride, 
does not lead to any bridging of $\mathrm{Au}$ nanoparticles. A solution with only PSS and Au nanoparticles, in identical concentrations, shows no signs of bridging between of Au particles. Also, the subsequent addition of $\mathrm{Na} 2 \mathrm{~S}$ leaves the system unaltered.

We surmise that the amount of $\mathrm{Cd}^{2+}$ ions relative to the number of Au nanoparticles is critical for the formation of the necklace morphology. When the $\mathrm{Cd}^{2+}$ ions bound to the citrate ion $\mathrm{COO}^{-}$groups on the surface of $\mathrm{Au}$ nanoparticles, the particles become bipolar, which led to clustered linear aggregation (Figure S1). Reduced exfoliation and smaller cluster lengths compared to Figure 2a were attributed to the absence of the molecular scaffold (i.e., PSS). Bridging in the cluster leads to a coordination number of two for most particles. At high coverage, the morphology of the clusters becomes more compact, and eventually begins to precipitate. Thus, it is critical to maintain low coverage of $\mathrm{Cd}^{2+}$ ions to obtain linear clusters.

In conclusion we have presented a method to fabricate an electrically conducting network of 1D Au nanoparticle necklaces that exhibits remarkable coulomb blockade effects. A single electron effect is observed when the particles are cemented with CdS. We are investigating further the electrical properties of these necklaces, which could be generalized for use with other nanoparticles, using surface reactions of carefully regulated precursor ion bridges. Such systems could be important for applications in nanoelectronic devices and sensors.

\section{Experimental}

Cadmium perchlorate hydrate solution $\left(1 \mathrm{mg} \mathrm{mL}^{-1}\right)$ was prepared and sonicated for $2 \mathrm{~h}$. A solution of $10 \mathrm{~nm}$ Au nanoparticles with $5.7 \times$ $10^{12}$ particles $\mathrm{mL}^{-1}(\mathrm{pH}$ ) ) was purchased from BBI International. These particles were prepared by a process of citrate reduction that resulted in citrate ions being absorbed onto the Au surfaces, making them negatively charged. The Cadmium solution was added drop-wise under constant stirring to $\mathrm{Au}$ solution until a volume ratio of $1: 4$ for $\mathrm{Cd}(\mathrm{aq}): \mathrm{Au}(\mathrm{aq})$ was reached, giving a total volume of $5 \mathrm{~mL}$. The solution was then stirred for $12 \mathrm{hrs}$. The color of the solution transformed from wine red to violet blue, which corresponded to a shift in the SPR band from $525 \mathrm{~nm}$ for the Au nanoparticle solution to $\sim 620 \mathrm{~nm}$ with a shoulder at $525 \mathrm{~nm}$ (AuCd curve in Fig S5). $1 \mathrm{mg} \mathrm{mL}^{-1}$ PSS (molecular weight $=500000 \mathrm{Da}$ ) solution was made and stirred for $4 \mathrm{~h}$. $200 \mu \mathrm{L}$ of this solution was added to the $\mathrm{Au}-\mathrm{Cd}$ solution and stirred overnight. In the dark, 0.7 times the stoichiometric ratio with $\mathrm{Cd}, \mathrm{Na}_{2} \mathrm{~S}$ is added to this solution. This changed the absorbance spectrum significantly. The complete synthesis was conducted in inert nitrogen conditions. More details are available in the Supporting Information.
Cemented material (PSS-CdS-Au) was deposited by electrostatic attraction onto the oppositely charged substrate surface (cationic-functionalized) between Au electrodes, which were separated by $50 \mu \mathrm{m}$ gaps, on $\mathrm{SiO}_{2}$ and $\mathrm{Si}$ chips. The electrical measurements were performed by applying a bias $V$ across the electrode and the resultant current $I$ was recorded. $I-V$ data for different temperatures from the same sample are presented, hence no normalization was required. Similar qualitative results were obtained for other samples that were made using the same procedure.

\section{References}

[1] a) A. Bachtold, P. Hadely, T. Nakanishi, C. Dekker, Science 2001, 294, 1317. b) Y. Huang, X. Duan, Y. Cui, L. J. Lauhon, K. H. Kim, C. M. Lieber, Science 2001, 294, 1313.

[2] a) J. Kong, A. R. Franklin, C. Zhou, M. G. Chapline, S. Peng, K. Cho, H. Dai, Science 2000, 287, 622. b) J. B. Tok, F. Y. Chuang, M. C. Kao, K. A. Rose, S. S. Pannu, M. Y. Sha, G. Chakarova, S. G. Penn, G. M. Dougherty, Angew. Chem., Int. Ed. 2006, 45, 6900 .

[3] F. Patolsky, B. P. Timko, G. H. Yu, Y. Fang, A. B. Greytak, G. F. Zheng, C. M. Lieber, Science 2006, 313, 1100.

[4] M. Law, L. E. Greene, J. C. Johnson, R. Saykally, P. D. Yang, Nat. Mater. 2005, 4, 455.

[5] O. Harnack, W. E. Ford, A. Yasuda, J. M. Wessels, Nano Lett. 2002, 2, 919.

[6] R. Djalali, S. Y. Li, M. Schmidt, Macromolecules 2002, 35, 4282.

[7] Y. J. Kang, K. J. Erickson, T. A. Taton, J. Am. Chem. Soc. 2005, $127,13800$.

[8] A. N. Shipway, M. Lahav, R. Gabai, I. Willner, Langmuir 2000, $16,8789$.

[9] S. Minko, A. Kiriy, G. Gorodyska, M. Stamm, J. Am. Chem. Soc. 2002, 124, 10192.

[10] V. Maheshwari, R. F. Saraf, Langmuir 2006, 22, 8623.

[11] S. Lin, M. Li, E. Dujardin, C. Girard, S. Mann, Adv. Mater. 2005, 17, 2553

[12] Z. Y. Tang, N. A. Kotov, M. Giersig, Science 2002, 297, 237.

[13] S. Link, M. A. El-Sayed, J. Phys. Chem. B 1999, 103, 8410.

[14] J. P. Pantina, E. M. Furst, Langmuir 2006, 22, 5282.

[15] J. P. Wilcoxon, J. E. Martin, F. Parsapour, B. Wiedenman, D. F. Kelley, J. Chem. Phys. 1998, 108, 9137.

[16] N. Chestnoy, T. D. Harris, L. E. Brus, J. Phys. Chem. 1986, 90, 3393.

[17] Y. J. Hsu, S. Y. Lu, Langmuir 2004, 20, 23.

[18] W. A. Lopes, H. M. Jaeger, Nature 2001, 414, 735.

[19] K. K. Likharev, Proc. IEEE 1999, 87, 606.

[20] R. Parthasarathy, X. M. Lin, H. M. Jaeger, Phys. Rev. Lett. 2001, 87, 186807-1.

[21] R. Parthasarathy, X. M. Lin, K. Elteto, T. F. Rosenbaum, H. M. Jaeger, Phys. Rev. Lett. 2004, 92, 076801-1

We thank the National Science Foundation (NER 608877) for financial support and Dr. X.Z. Li, University of Nebraska-Lincoln, for help with TEM. 


\title{
ONLINE SUPPORTING INFORMATION FOR:
}

\section{Self-Assembly of Microns Long One Dimensional Network of Cemented Au Nanoparticles}

\author{
Vivek Maheshwari, Jennifer Kane, Ravi F. Saraf * \\ Department of Chemical Engineering, University of Nebraska - Lincoln \\ Lincoln, NE 68588, USA. \\ rsaraf@unlnotes.unl.edu
}

\section{SUPPORTING INFORMATION}

\section{I: Reagents \& Instruments:}

All reagents except for Au nanoparticles are purchased from Sigma-Aldrich and used without further purification. Distilled water filtered with Millipore $0.22 \mu \mathrm{m}$ filters (resistivity $>18 \mathrm{M} \Omega \mathrm{cm}$ ) is used for all synthesis reactions. Au $10 \mathrm{~nm}$ particles with anionic citrate functionality are purchased from BBI international.

UV-visible absorption spectra are recorded in an ocean-optics absorbance spectrophotometer equipped with $10 \mathrm{~mm}$ quartz cuvette holder for liquid sample. TEM analysis is performed in a Hitachi-H-9000 NAR and the samples are prepared by placing a drop of fresh solution on Carbon film coated Copper $(\mathrm{Cu})$ grids, followed by immediate removal of the excess solvent using a blotting paper. Photo-Luminescence spectra are recorded on a Hitachi spectrophotometer, F-4500.

\section{Safety}

It should be noted that Cadmium salts are carcinogenic and should be handled with proper care and precautions. 


\section{Methods}

A pH 6 solution of $10 \mathrm{~nm}$ Au nanoparticle of concentration $5.7 \times 10^{12}$ particles $/ \mathrm{ml}$ is purchased from BBI International. These particles are prepared by citrate reduction, resulting in citrate ions being absorbed on the Au surface making them negatively charged. The UV-Vis spectrum shows the well known surface plasmon resonance (SPR) absorption band at $\sim 525 \mathrm{~nm}$ (Au curve in Fig S5). Cadmium perchlorate hydrate solution of concentration $1 \mathrm{mg} / \mathrm{ml}$ (Cd solution) is separately prepared and sonicated for 2 hours and added drop wise to Au solution under constant stirring at a volume ratio of 1:4. The solution is allowed to stir for $12 \mathrm{hrs}$. The color of the solution is transformed from wine red to violet-blue that corresponds to a shift in the SPR band from $525 \mathrm{~nm}$ for $\mathrm{Au}$ nanoparticle solution to $\sim 620 \mathrm{~nm}$ with a shoulder at $525 \mathrm{~nm}$ (Au-Cd curve in Fig S5). A $1 \mathrm{mg} / \mathrm{ml}$ solution of PSS (molecular weight 500,000 Dalton) is made and stir for 4 hours. $200 \mu \mathrm{l}$ of PSS solution is added to the $\mathrm{Au} / \mathrm{Cd}$ solution and allowed to stir overnight. No significant change in absorbance spectrum is observed except for the appearance of the PSS absorbance at $265 \mathrm{~nm}$. Following this $\mathrm{Na}_{2} \mathrm{~S}$ is added to the solution in 0.7 times the stoichiometric ratio with respect to $\mathrm{Cd}$ in dark. The absorbance spectrum changes significantly (curve PSS-CdS-Au in Fig. S5). The complete synthesis is done under inert nitrogen conditions.

Energy Dispersive X-ray Analysis (EDX), elemental analysis is performed on the cemented necklace with a $60 \mathrm{~nm}$ beam spot size. The spectrum shows presence of Au and $\mathrm{CdS}$ in the cemented necklace (Fig S7). The $\mathrm{Cu}$ peak is due to the $\mathrm{Cu}$ grid used for sample preparation and the $\mathrm{Cr}$ peak is due to the sample holder of the TEM instrument.

Electrical measurement is done on Au electrodes $50 \mu \mathrm{m}$ apart on $\mathrm{SiO}_{2} / \mathrm{Si}$ chips. The cemented material (PSS-CdS-Au) is deposited by electrostatic attraction to oppositely charged surface (Cationic functionalized). The presented current-voltage results at different temperatures are from the same sample, hence requiring no normalization for comparison. Similar qualitative results are obtained from other samples made with identical procedure. 


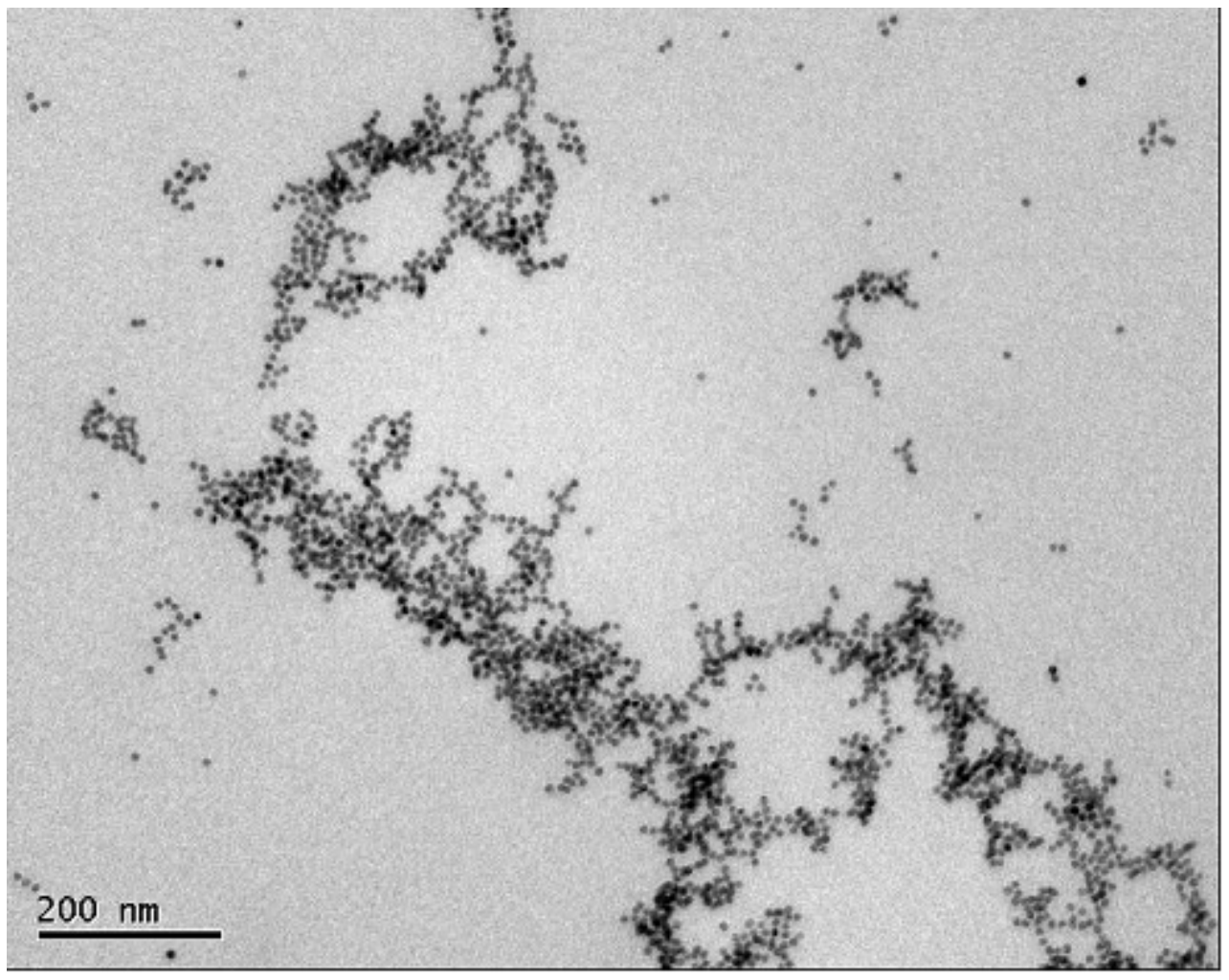

Fig S1. TEM analysis of the Au-Cd assembly. Clustered assembly of Au nanoparticles with string like morphology is observed.

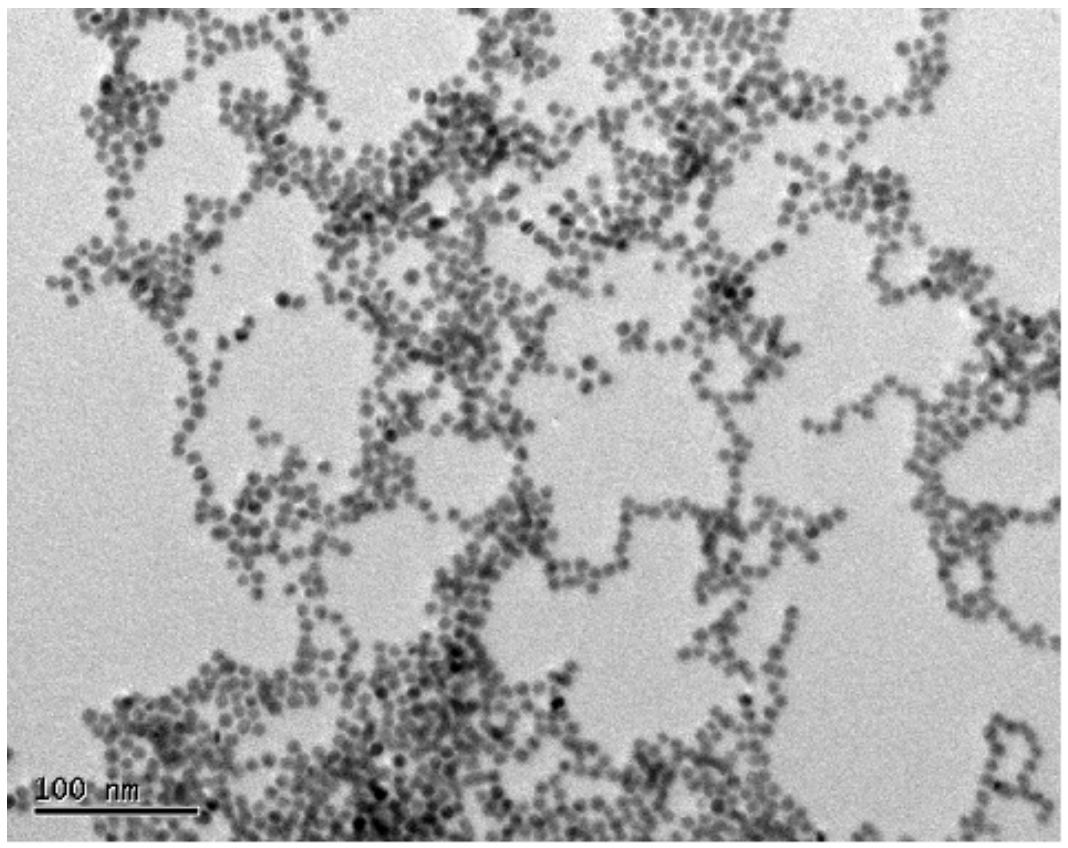

Fig S2. TEM analysis of the Au-Cd assembly. Clustered assembly of Au nanoparticles with string like morphology is observed. 


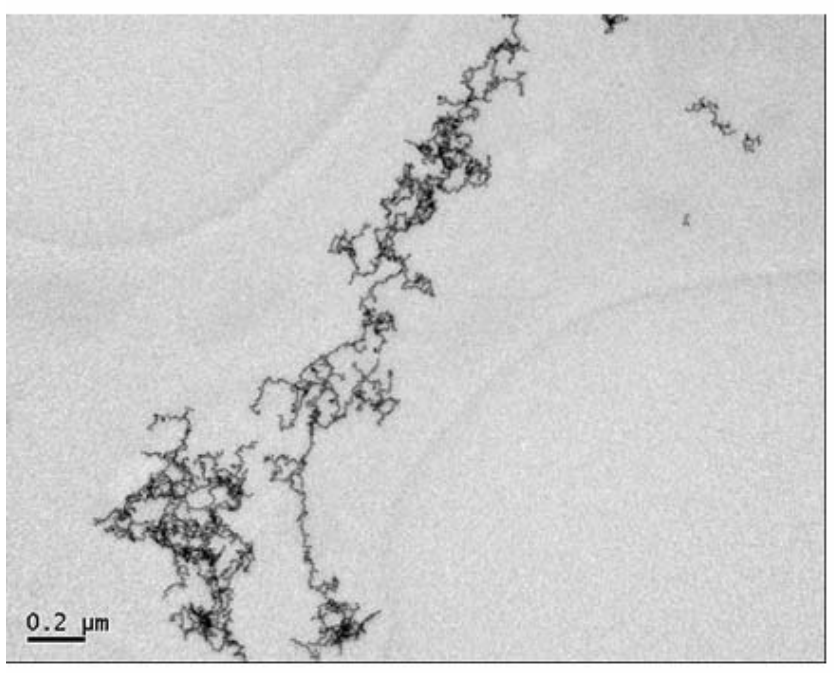

Fig S3. TEM analysis of the 1-D branched assemblies of discreet Au nanoparticles resulting from the PSS-Cd-Au interaction. The inset scale bars are $20 \mathrm{~nm}$.
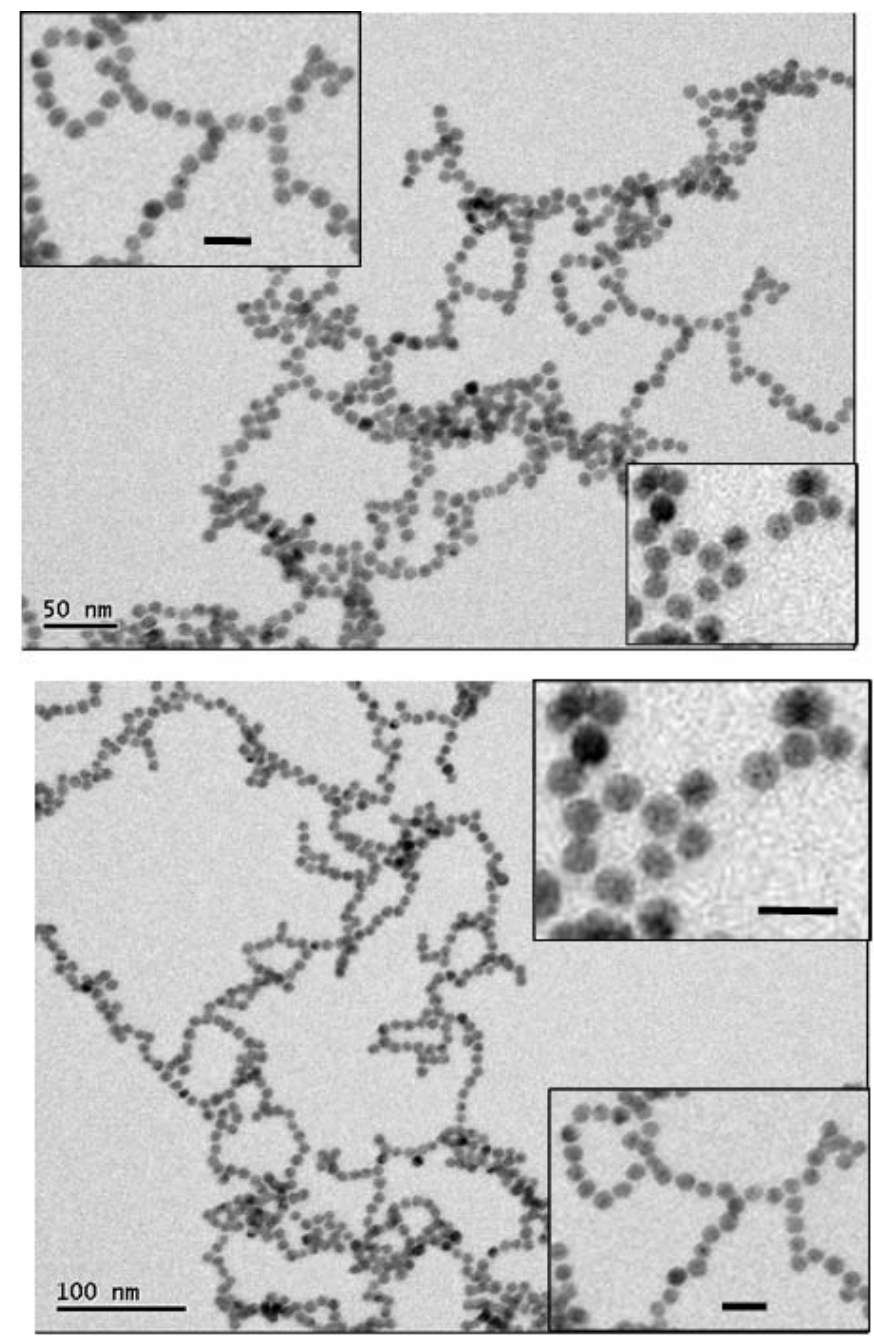


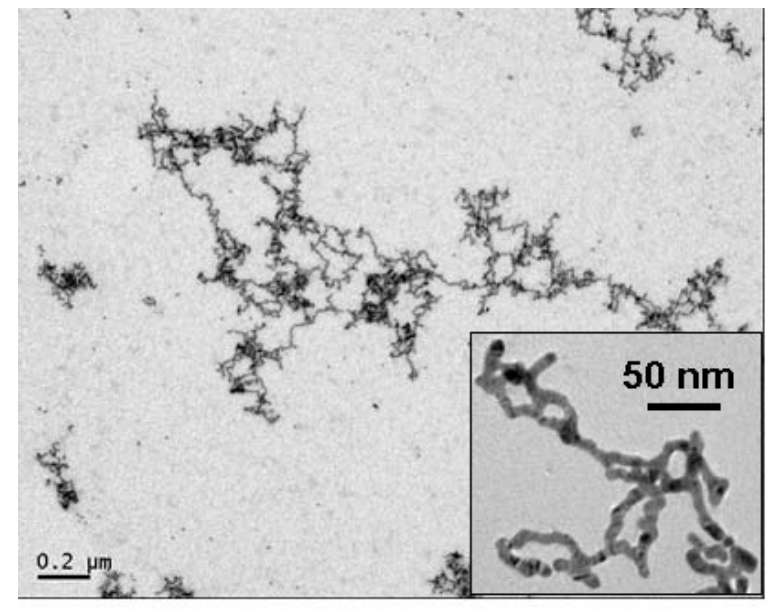

Fig S4. TEM analysis of the transformed assembly. On addition of $\mathrm{Na}_{2} \mathrm{~S}$ the discreet $\mathrm{Au}$ nanoparticles of fig S-3 are transformed to a continuous structure.
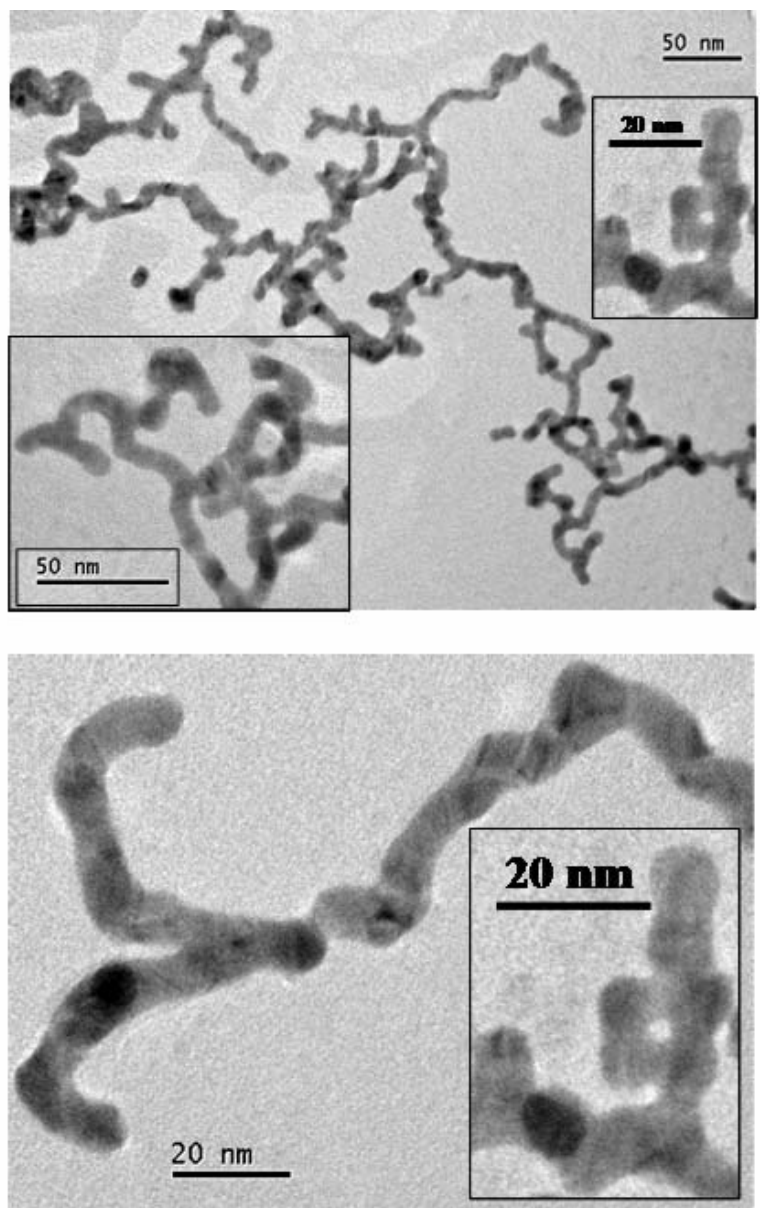


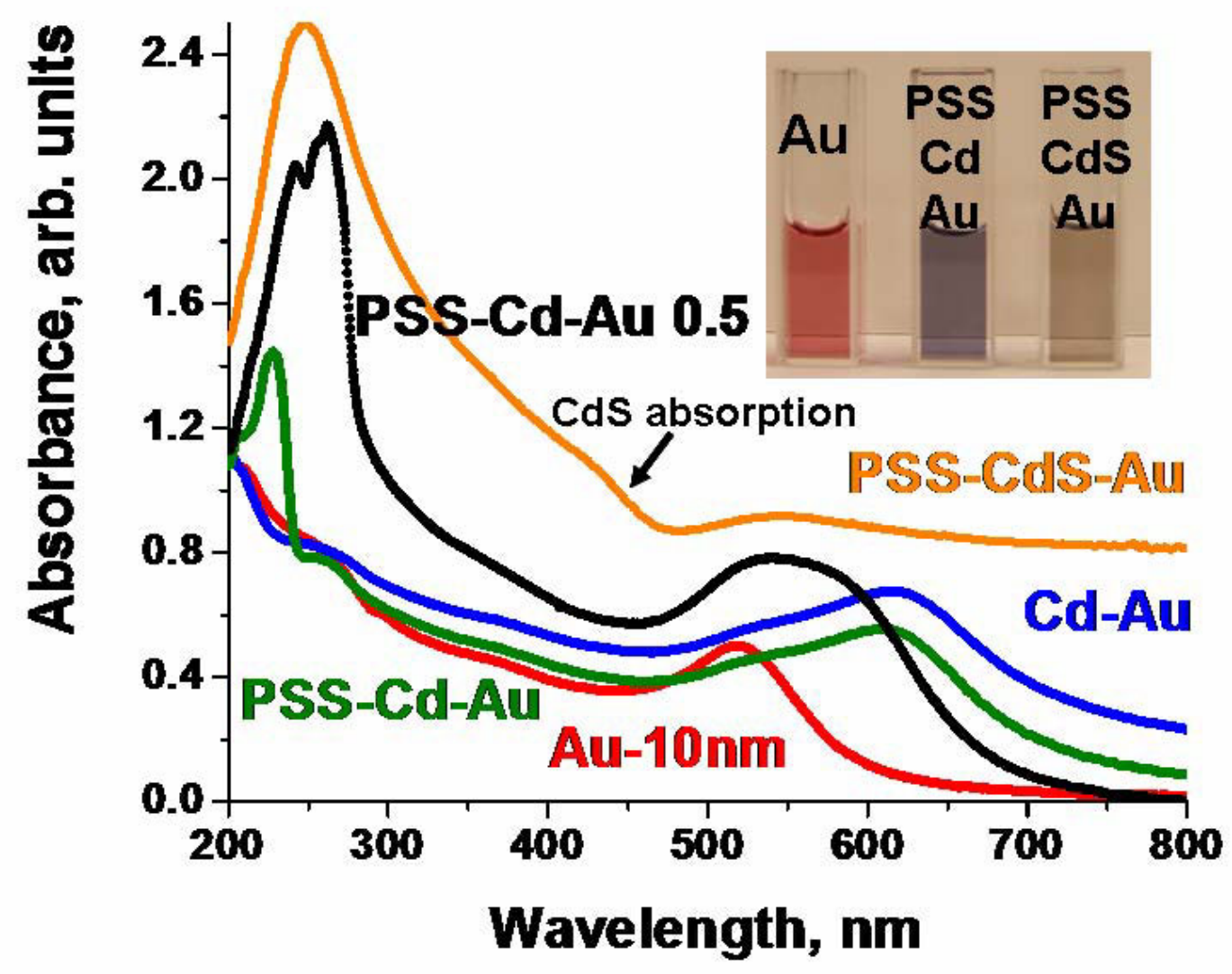

Fig S5. Absorption spectra of the synthesis at each stage of transformation. The PSS-CdSAu spectrum has been shifted vertically for better visualization. The change in the color of the solution from wine red to violet blue and to muddy yellow is visible in the photograph 


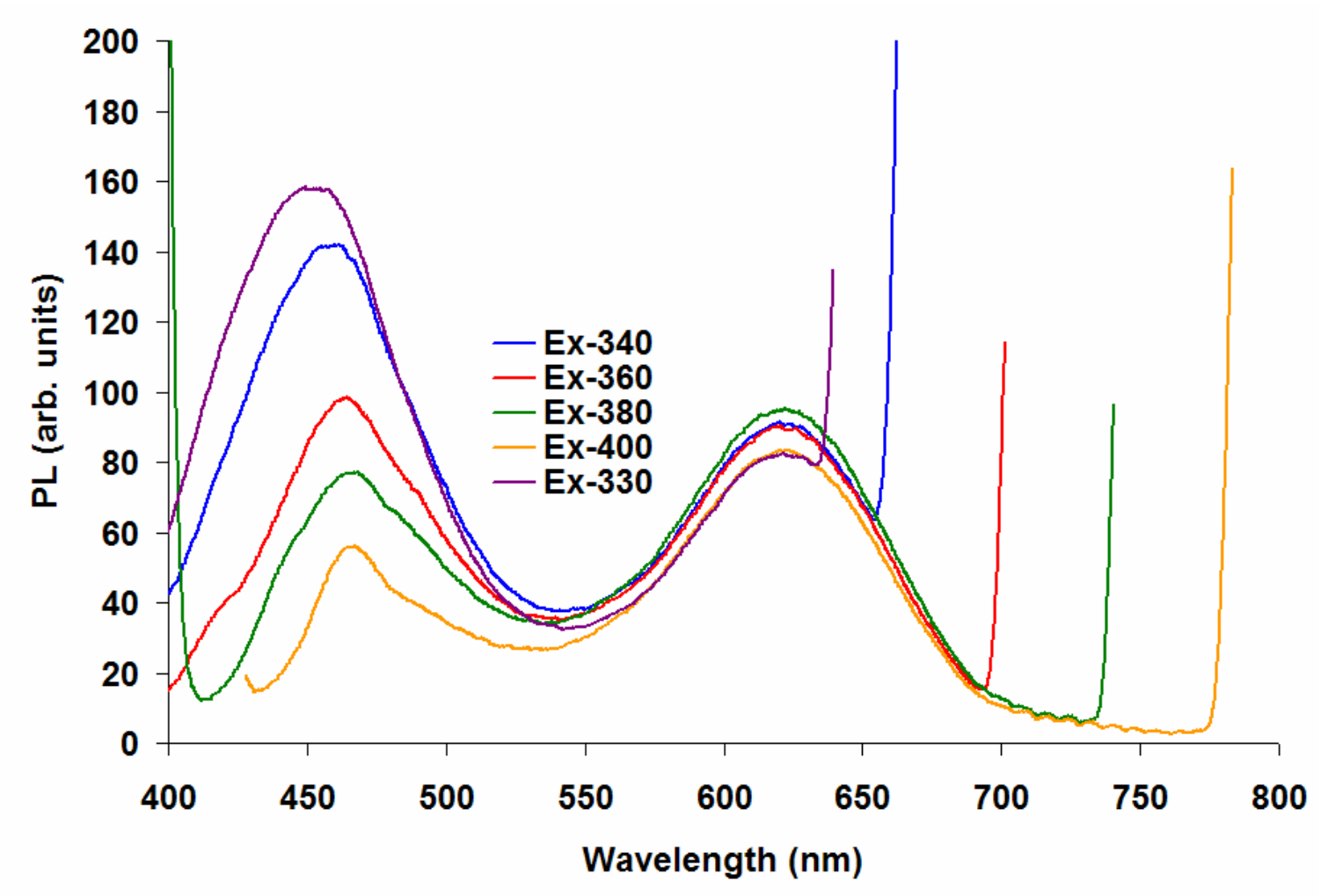

Fig S6. Photoluminescence emission spectra of the PSS-CdS-Au, 1-D branched continuous assembly. A new emission peak at $620 \mathrm{~nm}$ appears on addition of $\mathrm{Na}_{2} \mathrm{~S}$, due to formation of CdS. Inset shows the spectra for PSS-Cd-Au assembly, showing only the Au nanoparticle peak. A similar spectrum is shown by Au nanoparticles.

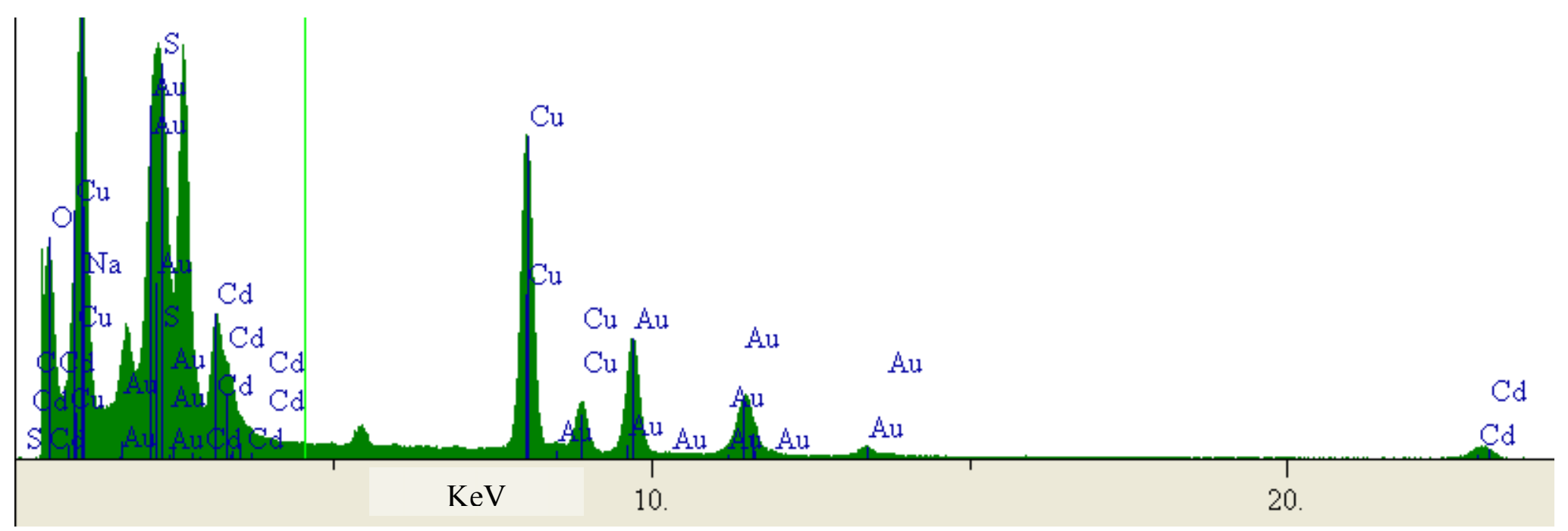

Fig S7. EDX spectra of the PSS-CdS-Au, 1-D branched continuous assembly. The collection is done using a $60 \mu \mathrm{m}$ beam spot size. The analysis shows the presence of $\mathrm{Au}, \mathrm{Cd}$ and $\mathrm{S}$ in the assembly. The $\mathrm{Cu}$ peak is due to $\mathrm{Cu}$ grids used for making the TEM sample. The peak at $\sim 5.7 \mathrm{KeV}$ is due to Chromium, the material of TEM sample holder. 


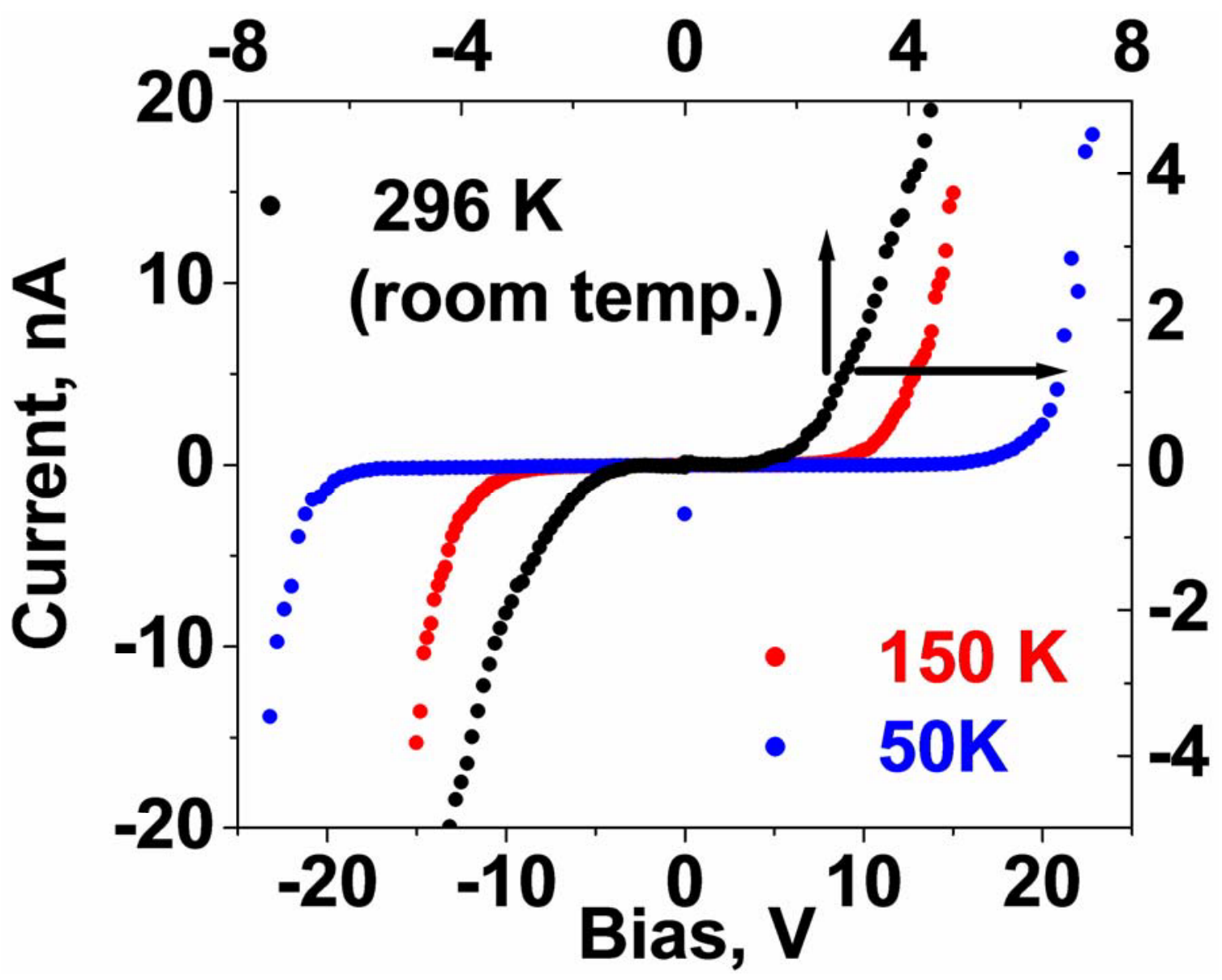

Fig S8. Electrical measurements of the PSS-CdS-Au, 1-D branched continuous assembly deposited between Au electrodes, $50 \mu \mathrm{m}$ apart. Remarkably high coulomb blockade is observed even at room temperature. The magnitude of blockade increases with temperature. 


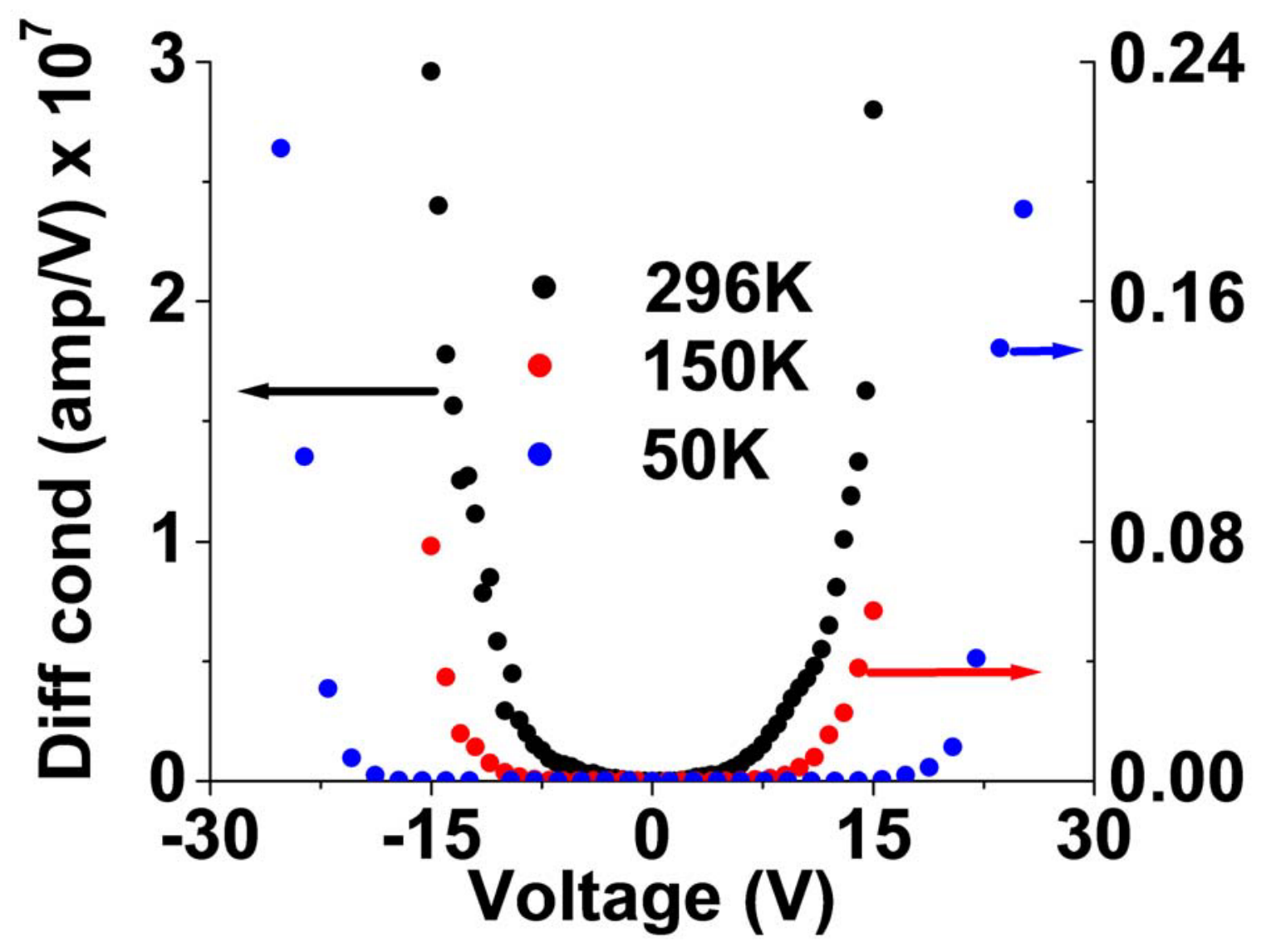

Fig S9. Differential conductivity plots from electrical measurements of the PSS-CdS-Au, 1-D branched continuous assembly deposited between Au electrodes, $50 \mu \mathrm{m}$ apart. Remarkably high coulomb blockade is observed even at room temperature. The magnitude of blockade increases with temperature. 